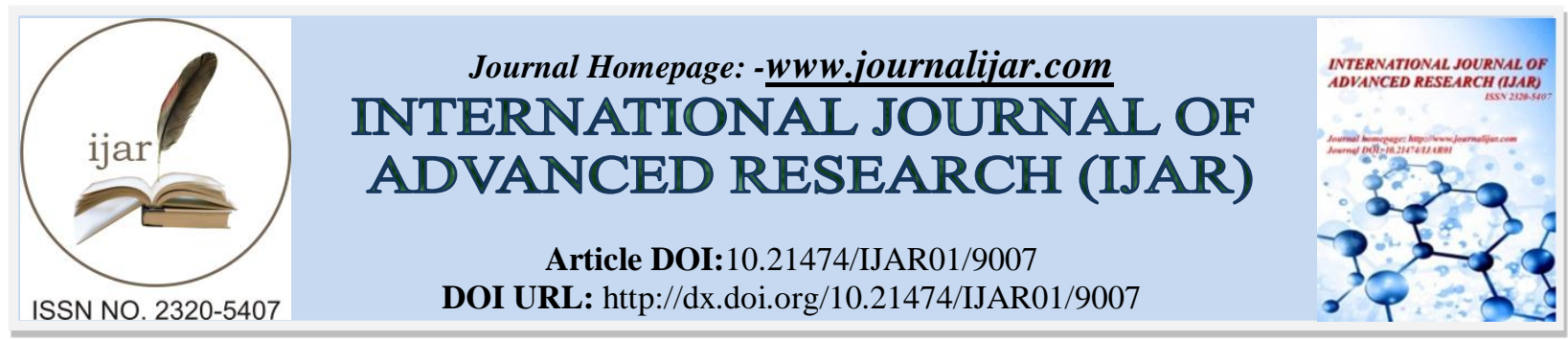

RESEARCH ARTICLE

\title{
NEOADJUVANT CHEMOTHERAPY BEFORE, CONCURRENTLY WITH AND AFTER RADIOTHERAPY FOR LOCALLY ADVANCED RECTAL CANCER.
}

\author{
Azza M Darwish ${ }^{1}$, Ahmed Abdel latif ${ }^{2}$ And Dina Abdallah ${ }^{3}$. \\ 1. Clinical Oncology Department, Faculty of Medicine, Alexandria University, Egypt. \\ 2. General Surgery Department, Faculty of Medicine, Alexandria University, Egypt. \\ 3. Pathology Department ,Faculty of Medicine, Alexandria University, Egypt.
}

\section{Manuscript Info}

\section{Manuscript History}

Received: 02 March 2019

Final Accepted: 04 April 2019

Published: May 2019

Key words:-

Rectal cancer, Locally advanced,

Neoadjuvant, Chemoradiotherapy,

Pathological complete response.

\begin{abstract}
Purpose: Neoadjuvant chemoradiotherapy (CRT) has a major impact in the local control of locally advanced rectal cancer (LARC). However, distant metastases is still a major challenge. One approach to address this issue is early administration of systemic chemotherapy. The purpose of this phase II study is to evaluate the feasibility and efficacy of short course of induction chemotherapy in LARC patients treated with standard preoperative CRT.

Methods and Mareials: Sixty-four patients with LARC were treated with two cycles of XELOX (capecitabine $1000 \mathrm{mg} / \mathrm{m}^{2}$ bid on days 1-14 and oxaliplatin $130 \mathrm{mg} / \mathrm{m}^{2}$ on day 1). One cycle of XELOX is given before CRT, while the second one is given in the resting period before surgery. During chemoradiation, blous 5-Fluorouracil $425 \mathrm{mg} / \mathrm{m}^{2}$ was dministered in the first 4 days and last 3 days of radiatation (50.4 Gy over 6 weeks ). Surgery was recommended 6 to 8 weeks after completion of CRT, followed by 4 four cycles of adjuvat XELOX. Results: Fifty-six patients underwent surgery; The pathological complete recponse (pCR) rate was seen in $16(28.6 \%)$ patients, R0 resection was achieved in $54(94.6 \%)$ patients. Tumor and nodal downstaging were observed in $40(71.4 \%)$ and $25(44.6 \%)$ patients, respectively. Sphincter preservation was achieved in 44(78.6\%) patients. Three year disease-free survival was $70.3 \%$, and overall survival was $82.8 \%$.

Conclusion: one cycle of XELOX before and after radiotherapy concurrent with 5-flourouracil for LARC results in a reasonable pCR, R0 resection and sphincter preservation, with acceptable safety and tolerability.
\end{abstract}

Copy Right, IJAR, 2019,. All rights reserved.

\section{Introduction:-}

Colorectal cancer ranks the fourth in worldwide cancer incidence and the second leading cause of cancer death. Cancer rectum represent about one third of cancer death of colorectal cancer. ${ }^{[1]}$ Preoperative fluorouracil and radiation followed by total mesorectal excision (TME) and four months of postoperative adjuvant fluorouracil is the standard treatment for stage II (T3,T4NO) and stage III (Tany N1/N2) rectal cancer,resulting in local relapse rate

Corresponding Author:-Azza M Darwish.

Address:-Clinical Oncology Department, Faculty of Medicine, Alexandria University, Egypt. 
less than $10 \%$ after 5 years ${ }^{[2]}$. The 5 - year distant relapse rate however is about $30 \%$ and continue to be a major challenge ${ }^{[3-5]}$.

Distant recurrence rates now exceed those of local recurrence, and this highlights the importance of delivering adequate systemic therapy for patients with locally advanced rectal cancer ( LARC) ${ }^{[5]}$.

Unfortunately, several trials have shown that nearly $50 \%$ of patients with LARC who are treated with preoperative chemoradiotheray (CRT) followed by surgery, are unable to complete the planned dose of adjuvant chemotherapy, and about 30\%are unable to receive any adjuvant chemotherapy.This suboptimal compliance to adjuvant chemotherapy is attributed to toxicities,surgical complications, or patients refusal especially after achieving good response to CRT ${ }^{[6,7]}$.

One strategy to adress this poor compliance, is to deliver induction chemotherapy before preoperative CRT. Induction chemotherapy may be associated with better treatment compliance and allow full systemic doses of chemotherapy to be delivered. Other advantages of induction chemotherapy is early eradication of distant micrometastasis, the possibility of shrinking or downstaging a locally advanced tumor thereby facilitating more effective local treatment ${ }^{[8]}$.

One more advantage of induction chemotherapy is to overcome any delay in starting CRT and to overcome the absence of treatment in the resting period (between end of CRT and surgery).Theoreticaly there might be a risk of tumor progression in these gaps ${ }^{[9,10]}$.

Several trials had studied the use of induction chemotherapy before the preoperative chemoradiation with different results obtained ${ }^{[11-17]}$, but we can conclude from these trials that induction chemotherapy is feasible, tolerable and did not affect the compliance to chemoradiotherapy or surgery ${ }^{[17]}$.

In order to enhance the systemic control, overcome delay of initiation of chemoradiation and to improve tolerance to systemic chemotherapy, we conducted this prospective phase II study of induction chemotherapy. The aim of this trial is to assess the feasibility, efficacy (in term of response rate) and toxicities of short course of induction chemotherapy given before surgery in patients with LARC.

This prospective single arm phase II study was carried out on 64 patients with LARC who presented to Clinical Oncology and Nuclear Medicine department, Alexandria University hospital in Egypt during the period between 2014 and 2016.

\section{Methods:-}

\section{Patient population}

Patients entering the study had histologically confirmed rectal adenocarcinoma. With inferior margin within $12 \mathrm{~cm}$ from the anal verge. Evidence of T3 or T4 disease with or without perirectal nodal involvement by magnetic resonance imaging (MRI) of the pelvis was required. Further inclusion criteria were an Eastern Cooperative Oncology Group (ECOG) performance status $0-2$ and adequate haematological, renal and liver functions (neutrophils $\geq 1500$, platelet count $\geq 100,000$, creatinine clearance $\geq 30 \mathrm{ml} / \mathrm{dl}$, total bilirubin $\leq 2$ times the upper limit of normal limit and liver transaminases $\leq 3$ times the upper normal limit ).

Patients aged more than 18 years and both sex were included.

Exclusion criteria were metastatic disease, previous chemotherapy for colorectal cancer or prior radiotherapy to the pelvis, history of another malignancy within the last 5 years, any contraindication to radiotherapy, clinically significant cardiac disease, malabsorption syndrome, peripheral neuropathy > grade1 according to National Cancer Institute Common Terminology Criteria for Adverse Events (NCI CTC version 3.0) Pregnant or lactating woman were excluded.

All patients provided written informed consent. The study protocol was approved by a local independent ethics committee and conducted in accordance with the Declaration of Helsinki 


\section{Pretreatment evaluation}

Before study entry, all patients. were required to have complete history taking, physical examination including digital rectal examination Complete laboratory tests included a full blood count, creatinine, liver transaminases, alkaline phosphatase, total bilirubin and carcinoembryonic antigen (CEA) measurement., computed tomography of the chest, abdomen and MRI pelvis.

\section{Treatment protocol: \\ Radiation therapy:}

ALL Patients underwent CT simulation in the prone position, (A full bladder protocol is used for planning and treatment as this displaces small bowel superiorly. A radio-opaque marker is placed on the anal verge and oral contrast was used to help identify small bowel. A planning CT scan was performed with $3 \mathrm{~mm}$ slices from the level of $\mathrm{L} 5$ to $2 \mathrm{~cm}$ below the anal marker.

The gross tumor volume (GTV) included all gross tumor seen on the planning CT scan with reference to information from diagnostic endoscopy, MRI and DRE. Any involved lymph nodes, extrarectal extension, or extranodal deposits seen on MRI should be included.

The clinical target volume (CTV) included the GTV, rectum and lymph node regions, including, mesorectum, precacral nodes, internal iliac nodes, and superior rectal node.

The external iliac lymph nodes are included if there was anterior organ involvement. The PTV was created by adding a margin of $1-1.5 \mathrm{~cm}$ to the CTV to account for daily setup error and organ motion. A boost to the primary tumor was added. The phase $2 \mathrm{PTV}$ then included the tumor mass with a $2 \mathrm{~cm} 3 \mathrm{D}$ margin.

High energy beams (6-15 MV) were used, a three- or four-beam arrangement includes a one direct posterior beam and two lateral wedged beams depending on the shape of the patient. With advanced disease where there is bladder or anterior abdominal wall involvement, a four-beam arrangement or AP opposing beams may need to be used to increase the dose anteriorly.

\section{Dose - fractionation:}

In phase 1 patients were treated with 45 Gy in 25 daily fractions of 1.8 Gy given in 5 weeks then phase 2 patients were given $5.4 \mathrm{~Gy}$ in 3 daily fractions of $1.8 \mathrm{~Gy}$.

The plans were optimized to deliver $95 \%$ of the prescribed dose to at least $95 \%$ of the PTV.

\section{Dose constrains:}

The dose constrains to small bowel was up to $45 \mathrm{~Gy}$, while the femoral head and neck up to $45 \mathrm{~Gy}$ and the bladder V40 $<50 \%$.

\section{Neoadjuvant chemotherapy:}

Patients received one cycle of neoadjuvant chemotherapy XELOX regimen (oxaliplatin $130 \mathrm{mg} / \mathrm{m}^{2}$ day 1 plus capcitabine $1000 \mathrm{mg} / \mathrm{m}^{2}$ twice daily on day 1 to 14) 3 weeks before radiation therapy. During radiotherapy blous 5Fluorouracil $425 \mathrm{mg} / \mathrm{m}^{2}$ was dministered in the first 4 days of the first week and 3 days in the fifth week, one week after completion of chemoradiotherapy patients received one additional cycle of chemotherapy XELOX.

\section{Assessment of response and toxicities:}

Patients in the current study were followed before each cycle of chemotherapy and weekly during radiotherapy to assess the signs and symptoms of toxicity. Adverse events were recorded using Common Terminology Criteria for Adverse Events (CTC)version 4.0 $0^{[18]}$.

One week after completion of CRT, all patients were subjected to complete physical examination, digital rectal examination.

MRI pelvis and CT chest, abdomen and pelvis were done after 4 weeks from the end of CRT to assess the response radiologically and to rule out tumor progression. 


\section{Surgery:}

Total mesorectal excision (TME) was performed at $6-8$ weeks after completion of CRT the choice of surgical procedure (abdomino perineal or anterior resection) was at the surgical discretion.

\section{Adjuvant chemotherapy:}

Adjuvant chemotherapy with XELOX was given for 4 cycles at $3-6$ weeks after surgery. Oxaliplatin $130 \mathrm{mg} / \mathrm{m}^{2}$ was given on day 1 plus capcitabine $1000 \mathrm{mg} / \mathrm{m}^{2}$ twice daily on day 1 to 14 ) every 3 weeks.

\section{Pathology:}

After TME, tissue specimens from patients receiving neoadjuvant chemoradiation were thoroughly sectioned, with careful examination of the tumor site. In cases of residual macroscopic tumor, standard pathologic examination was performed on 3 to 5 sections to investigate the deepest invasion in the bowel wall. If no macroscopic tumor was present and only a small ulcer was observed, the ulcer with a 2-cm margin was examined for residual tumor and deepest invasion in the bowel wall, and the circumferential resection margin was measured. Pathologic complete response (pathCR) was defined as the complete disappearance of all tumor cells in the primary lesion or sampled lymph nodes ${ }^{[19]}$.

Tumor regression was assessed only in the primary tumor; lymph node metastases were not included in the assessment. Acellular pools of mucin in specimens from patient receiving neoadjuvant therapy was considered to represent completely eradicated tumor and was not used to assign pT stage or counted as positive lymph nodes.

Assessment of pathological response was based on: 1) residual tumor cells and 2) tissues replacing tumor cells in areas where the tumor had regressed. These "replacement" tissues might be fibrotic, inflammatory; acellular mucin pools, or, occasionally, necrosis and calcifications. ${ }^{[20]}$ When classifying rectal cancer response to chemoradiation, the AJCC Staging Manual (7th edition) system was used ${ }^{[21]}$.

All mesenteric tissue was manually dissected and examined for nodes. Fat-clearing techniques were not used. If fewer than 12 nodes were identified on first examination, a second attempt to locate lymph nodes was performed. This was performed in the standard fashion, with careful palpation and sectioning through fatty tissues. Submission of additional sections of mesorectum was based on the impression of gross examination; any tissue suspicious for being a lymph node was submitted. All grossly negative or equivocal lymph nodes were submitted entirely. Grossly positive lymph nodes were partially submitted for microscopic confirmation of metastasis ${ }^{[22]}$.

\section{Statistical analysis}

Categorical variables are presented as fre-quencies (percentages) and continuous variables as means with standard devia-tions or medians with ranges. Statistical analyses were performed with SPSS sta-tistical software version 15 for Windows (SPSS Inc., Chicago, IL, USA). Disease-free survival (DFS) was defined as the time from date of trial entry until disease progression, relapse, or death from any cause. Overall survival (OS) was calculated from the date of trial entry until death from any cause or was censored at last follow-up. Both DFS and OS were estimated by using the Kaplan-Meier methods.

\section{Results:-}

\section{Clinicopathological features:}

Between 2014 and 2016, 64 patients with advanced rectal cancer were enrolled in this phase II prospective study Table 1 presents the clinicopathological features of the patients included in this study.

The median age at diagnosis was 49 years old (range 29_68years). Fifty (78.12\%) patients were males and fourteen $(21.8 \%)$ were females. All patients had a good performance status according to ECOG, with 29(45.3\%) having performance status 0 and $35(54.7 \%)$ having performance status 1 . Thirty-four $(53.1 \%)$ patients had a tumor located $5 \mathrm{~cm}$ or less from the anal verge. The range of distal tumor margin from anal verge was $3-12 \mathrm{~cm}$, with a median of 5 $\mathrm{cm}$. The predominant clinical stage was CT3, 78.1\% of the patients, and clinically positive lymph nodes was found in $71.9 \%$ of the patients. 


\section{Compliance and acute toxicities:}

Neoadjuvant chemotherapy: Fifty nine (92.2\%) patients received the planned 2 cycles of XELOX, while 5 patients received only one cycle due to lost follow up in 4 patients during the CRT, and refusal of the fifth patient after developing grade 3 diarrhoea during the CRT.

Neoadjuvant CRT: Sixty (93.8\%) patients received full dose of radiation. Four patients discontinued radiotherapy after developing grade 3 diarrhoea and lost follow up. Eight patients (13.3\%) had treatment gaps ranging from 3 to 10 days due to grade 3 gastrointestinal toxicities. Gap correction was done for patients who skipped 5 fractions or more.

Surgery: TME was performed in 56 patients only. Eight of our patients did not proceed to surgery; due to lost follow up of four patients during the concomitant chemoradiotherapy after developing grade 3 diarrhoea, death of one patient due to grade 3 neutropenia after the second cycle of neoadjuvant chemotherapy, and reluctancy to undergo surgery after achieving complete clinical response in three other patients.

The median interval time from the end of CRT to surgery was 57 days (Range 48- 73 days). Spincter-saving surgery was done in 44 patients (78.6\%), while abdominoperineal resection was done in 12 patients (21.4\%). Microscopically clear resection margin (R0) was achieved in $54(94.6 \%)$ patients. While in the remaining two patients, one had involved radial margin and the other had involved distal margin.

Four patients had wound infection requiring antibiotics, one patient had pelvic abscess requiring drainage, one patient had anastomotic leak requiring reoperation, one patient had obstruction /ileus, while delayed wound healing occurred in 3 patients. No postoperative mortalities occurred in this study.

Adjuvant chemotherapy: Fifty- six patients started adjuvant chemotherapy. Fifty- three (94.6\%) patients completed the planned course of adjuvant chemotherapy while 3 patients did not due to grade 3 peripheral neuropathy in 2 patients and grade 3 neutropenia in the third one. Median time from surgery to adjuvant chemotherapy was 30 days (range 21-43 days).

Acute toxicities: The most common adverse events during neoadjuvant treatment (Neoadjuvant XELOX and CRT) are listed in Table 2.The most frequently occurring grade 3/4 nonhaematological adverse event was diarrhoea $(11.7 \%)$, followed by proctatitis $(1.7 \%)$. As regards haematological adverse events, no grade $3 / 4$ toxicities except for one patient (1.6\%) who developed grade 3 leucopenia and died after receiving the second cycle of induction XELOX.

During adjuvant chemotherapy, two patients (3.6\%) developed grade 3 neuropathy. Grade 3 leucopenia was reported in one patient (1.8\%). The most common adverse events during adjuvant chemotherapy are listed in Table 2.

\section{Efficacy parameters:}

Sixteen patients (28.6\%) achieved complete pathological response (ypT0N0).Near complete pathological response (ypT1N0) was found in eleven patients $(19.6 \%$ ), (Figure 1,2). Details of surgical and pathologic findings are present in Table 3. By comparing baseline clinical staging with final pathological staging (Table 4) it was found that tumor downstaging was achieved in 40 patients $(71.4 \%)$ and nodal downstaging was achieved in 25 patients (44.6\%).

After a median follow-up of 30 months, the 3-year disease free survival rate was $70.3 \%$ (95\% CI, 62\% to 91\%) (Figure 3) and the 3-year overall survival rate was $82.8 \%$ (95\% CI, 77\% to 96\%). (Figure 4)

\section{Discussion:-}

The gold standard treatment for stage II (T3-T4,N0) and stage III (T any, N1,N2) rectal cancer is neoadjuvant concurrent chemoradiation, followed by surgery (TME) then adjuvant chemotherapy given after surgery ${ }^{[23-25]}$.

Most recurrences after this treatment paradigm are distant recurrence, this may be due to the delay in delivery of systemic chemotherapy which allows for growth of distant micrometastases that may already exist. However, if systemic chemotherapy administered early, this may allow a higher likelihood of early eradication of micrometastases. Morever, the ability to administer full systemic doses of chemotherapy and the compliance of the patients to treatment, may be improved if chemotherapy is given before surgery ${ }^{[26]}$. 
There are different ways of delivering neoadjuvant chemotherapy, with no randomized trials comparing the types of regimens or number of cycles.

Our study has shown the tolerability and feasibilty of giving one cycle of XELOX regimen before and after CRT in locally advanced rectal cancer. We preferred to limit the number of neoadjuvant chemotherapy to two cycles only, to avoid delaying the primary surgery for a longer period.

Some studies, that give more than two cycles of chemotherapy have shown that progression during induction chemothrapy although rare, but remains possible, as shown by Chau et al, wherein $12 \%$ of patients who were treated with four cycles of induction XELOX did not show any tumor control ${ }^{[27]}$.

In our study, complete pathological response was achieved in $28.6 \%$ and a near complete pathological response in $19.6 \%$ of our patients. This response rate is in accordance with earlier studies using induction chemotherapy followed by CRT ${ }^{[12-16]}$.

Chau et al ${ }^{[27]}$, showed that $24 \%$ of patients with LARC achieved pCR after four cycles of neoadjuvant capecitabine and oxaliplatin before CRT with capecitabine.

Cerek et al ${ }^{[26]}$, also reported, in his retrospective study, that the overall complete pCR was $29 \%$ in patients with LARC who underwent TME after recieving induction mFOLFOX6 chemotherapy then radiotherapy concomitant with either 5-FU or capecitabine.

However our results differ from that of Gao et al. ${ }^{[28]}$, who reported that $42.2 \%$ of patients achieved pCR. This may be attributed to the addition of oxaliplatin to the concurrent chemoradiotherapy. In contrary to our study, where we used Xeloda only concomitant with radiotherapy.

The benefit of adding oxaliplatin to conventional CRT remains controversial, some studies have found no benefit from adding oxaliplatin during CRT but found that grade 3/4 diarrhea increased from $4 \%$ with 5- fluorouracil to $15 \%$ with oxaliplatin +5 - fluorouracil ${ }^{[29]}$

On the other hand, a meta-analysis showed that addition of oxaliplatin might increase pCR rate and decreased metastatic rate ${ }^{[30]}$.

In our study we have chosen to give 5- fluorouracil only as a concurrent therapy, for fear of significant increase in toxicities and decreased tolerance of radiation if oxaliplatin is added.

Further studies are needed to determine if the benefit of adding oxaliplatin to conventional CRT outweigh the anticipated toxicities.

In our study, tumor downstaging was seen in $71.4 \%$ of our patients which are consistent to findings by Calvo et al.

${ }^{[31]}$ who reported $75 \%$ of tumor downstaging after two cycles of induction FOLFOX 4 and Tegafur combined with RT. Schou et al. ${ }^{[12]}$ also reported $69 \%$ of tumor downstaging after neoadjuvant capecitabine and oxaliplatin (CAPOX) followed by RT concomitant with capecitabine.

Furthermore, nodal downstaging was achieved in $44.6 \%$ of our patients who present with radiologically positive lymph nodes, our results compare favourably with that of Koeberle et al. ${ }^{[16]}$ who reported $48 \%$ nodal downstaging, and Calvo et al ${ }^{[31]}$ who reported $40 \%$ nodal downstaging. However, other studies have shown higher rates of nodal downstaging. Finn et al. ${ }^{[32]}$ stated that downstaging in lymph nodes was obsreved in $60 \%$ of patients with LARC, treated with 3 cycles of induction CAPOX and raditherapy concurrent with CAPOX.

In our study, we showed that giving two cycles of induction chemotherapy added to induction CRT in patients with locally advanced rectal cancer resulted in an acceptable pCR, tumor and nodal downstaging. Pathological complete tumour response is a reliable surrogate for tumour control and can predict improved outcome ${ }^{[33]}$. Furthermore, eradication of metastasis from lymph nodes reflects the impact of effective neoadjuvant treatment and enhance $\operatorname{prognosis}^{[34,35]}$. 
In an exploratory analysis performed in 385 patients treated in the CAO/ARO/AIO-94 trial (preoperative chemoradiation arm) 5 year disease-free survival was significantly higher in patients with pCR, compared to patients with minor tumor regression $(86 \% \text { vs } 63 \%)^{[36]}$.

Total mesorectal excision (TME) was done for all of our patients who proceeded to surgery, with clear resection margin achieved in $94.6 \%$ of our patients.

Anterior resection was done in patients (78.6\%) and abdominoperineal resection in patients only $(21.4 \%)$ inspite of $53 \%$ of patients had low-lying tumours $(0-5 \mathrm{~cm}$ from anal verge $)$.

The most common toxicities encountered in our trial were gastrointestinal toxicities. Most of these toxicities were grade $1 / 2$ with the exception of grade 3 diarrhoea that occurred in $11.7 \%$ and grade 3 proctatitis in $1.7 \%$ of our patients during CRT. The most common haematological toxicity was neutropenia, two of our patients developed grade 3 neutropenia; one after induction chemotherapy and one after adjuvant chemotherapy.

We conclude that one cycle of XELOX before and after radiotherapy concurrent with 5-flourouracil is feasible and tolerable without compromizing the preoperative CRT completion and results in a reasonable pCR, R0 resection and sphincter preservation in patients with locally advanced rectal cancer.

\section{Disclosure}

The authors have stated that they have no conflict of interest

Table 1:-Patients and tumor characteristics $(\mathrm{N}=64)$

\begin{tabular}{|c|c|}
\hline Characteristics & No. $(\%)$ \\
\hline \multicolumn{2}{|l|}{ Age (years) } \\
\hline Median & 49 \\
\hline Range & $(29-68 \%)$ \\
\hline \multicolumn{2}{|l|}{ Gender } \\
\hline Male & $50(78.2 \%)$ \\
\hline Female & $14(21.8 \%)$ \\
\hline \multicolumn{2}{|l|}{ Performance status (ECOG) } \\
\hline 0 & $29(45.3 \%)$ \\
\hline 1 & $35(54.7 \%)$ \\
\hline \multicolumn{2}{|c|}{ Distance of tumor from anal verge $(\mathrm{cm})$} \\
\hline $0-5 \mathrm{~cm}$ & $34(53.1 \%)$ \\
\hline $5.1-10 \mathrm{~cm}$ & $20(31.3 \%)$ \\
\hline $10.1-12 \mathrm{~cm}$ & $10(15.6 \%)$ \\
\hline Range & $3-12 \mathrm{~cm}$ \\
\hline Median & $5 \mathrm{~cm}$ \\
\hline \multicolumn{2}{|l|}{ Pretreatment (CEA) level } \\
\hline $\mathrm{CEA}<5 \mathrm{ng}$ & $46(71.9 \%)$ \\
\hline $\mathrm{CEA}>5 \mathrm{ng}$ & $18(28.2 \%)$ \\
\hline \multicolumn{2}{|l|}{ Histological grading } \\
\hline Well differentiated & $2(3.1 \%)$ \\
\hline Moderate differentiated & $22(34.4 \%)$ \\
\hline Poorly/undifferentiated & $40(62.5 \%)$ \\
\hline \multicolumn{2}{|l|}{ Clinical T stage } \\
\hline c T3 & $50(78.1 \%)$ \\
\hline c T4 & $14(21.9 \%)$ \\
\hline \multicolumn{2}{|l|}{ Clinical N stage } \\
\hline c N0 & $18(28.1 \%)$ \\
\hline $\mathrm{c} \mathrm{N}_{+}$ & $46(71.9 \%)$ \\
\hline Median tumor size & $6 \mathrm{~cm}$ \\
\hline
\end{tabular}

Abbreviations: $\mathrm{ECOG}=$ Eastern Cooperative Oncology Group, $\mathrm{CEA}=$ carcinoembryonic antigen 
Table 2:-Most frequently reported treatment-related acute toxicities

\begin{tabular}{|c|c|c|c|}
\hline Acute toxicity & Grade 1 no. (\%) & Grade 2 no. (\%) & Grade 3 no. (\%) \\
\hline \multicolumn{4}{|c|}{ During neoadjuvant chemotherapy $(n=64)$} \\
\hline \multicolumn{4}{|l|}{ (Haematological toxicity) } \\
\hline Anaemia & 3 pts $(4.7 \%)$ & 0 & 0 \\
\hline Leukopenia & 5 pts $(7.8 \%)$ & $1 \mathrm{pt}(1.6 \%)$ & $1 \mathrm{pt}(1.6 \%)$ \\
\hline Thrombocytopenia & 0 & 0 & 0 \\
\hline \multicolumn{4}{|c|}{ (Non-haematological toxicity) } \\
\hline Nausea and vomiting & 12 pts $(18.8 \%)$ & 5 pts $(7.8 \%)$ & 0 \\
\hline Neuropathy & 0 & 0 & 0 \\
\hline Diarrhoea & 5 pts $(7.8 \%)$ & 2 pts $(3.1 \%)$ & 0 \\
\hline Stomatitis & 0 & 0 & 0 \\
\hline Hand-foot syndrome & 0 & 0 & 0 \\
\hline \multicolumn{4}{|c|}{ During neoadjuvant chemoradiotherapy $(\mathrm{n}=60)$} \\
\hline Dermatitis & 13 pts $(21.6 \%)$ & 11 pts $(18.3 \%)$ & 0 \\
\hline Procatitis & 8 pts $(13.3 \%)$ & 17 pts $(28.3 \%)$ & $1 \mathrm{pt}(1.7 \%)$ \\
\hline Diarrhoea & 12 pts $(20 \%)$ & 9 pts $(15 \%)$ & 7 pts $(11.7 \%)$ \\
\hline \multicolumn{4}{|c|}{ During adjuvant chemotherapy $(n=56)$} \\
\hline \multicolumn{4}{|l|}{ (Haematological toxicity) } \\
\hline Anaemia & 6 pts $(10.7 \%)$ & 3 pts $(5.6 \%)$ & 0 \\
\hline Leukopenia & 13 pts $(23.2 \%)$ & 7 pts $(12.5 \%)$ & $1 \mathrm{pt}(1.8 \%)$ \\
\hline Thrombocytopenia & 5 pts $(8.9 \%)$ & 0 & 0 \\
\hline \multicolumn{4}{|c|}{ (Non-haematological toxicity) } \\
\hline Nausea and vomiting & 8 pts $(14.3 \%)$ & 6 pts $(10.7 \%)$ & 0 \\
\hline Neuropathy & 14 pts $(25 \%)$ & 7 pts $(12.5 \%)$ & 2 pts $(3.6 \%)$ \\
\hline Diarrhoea & 3 pts $(5.4 \%)$ & $1 \mathrm{pt}(1.8 \%)$ & 0 \\
\hline Stomatitis & 5 pts $(8.9 \%)$ & 0 & 0 \\
\hline Hand-foot syndrome & 6 pts $(10.7 \%)$ & 0 & 0 \\
\hline
\end{tabular}

Table 3:-Surgical and pathological features $(n=56)$

\begin{tabular}{|c|c|}
\hline Features & No. $(\%)$ \\
\hline R0 resection of primary tumor & 54 pts $(96.4 \%)$ \\
\hline Median NO. of lymoh nodes (range) & $4(1-13)$ \\
\hline \multicolumn{2}{|l|}{ Types of surgery } \\
\hline APR & $12(21.4 \%)$ \\
\hline AR & $44(78.6 \%)$ \\
\hline \multicolumn{2}{|c|}{ The interval between end of CRT and surgery } \\
\hline Median & 57 days \\
\hline Range & (48-73 days) \\
\hline \multicolumn{2}{|l|}{ Pathologic response } \\
\hline pCR & 16 pts $(28.6 \%)$ \\
\hline Near complete response & 11 pts $(19.6 \%)$ \\
\hline \multicolumn{2}{|l|}{ Pathologic T stage } \\
\hline $\mathrm{yT}_{0}$ & 16 pts $(28.6 \%)$ \\
\hline $\mathrm{yT}_{1}$ & 11 pts $(19.6 \%)$ \\
\hline $\mathrm{yT}_{2}$ & 9 pts $(16 \%)$ \\
\hline $\mathrm{yT}_{3}$ & 16 pts $(28.6 \%)$ \\
\hline $\mathrm{yT}_{4}$ & 4 pts $(7.1 \%)$ \\
\hline \multicolumn{2}{|l|}{ Pathologic N stage } \\
\hline $\mathrm{yN}-\mathrm{ve}$ & 38 pts $(67.9 \%)$ \\
\hline $\mathrm{yN}+\mathrm{ve}$ & 18 pts $(32.1 \%)$ \\
\hline T downstaging & 40 pts $(71.4 \%)$ \\
\hline $\mathrm{N}$ downstaging & 25 pts $(44.6 \%)$ \\
\hline
\end{tabular}


Abbreviations: $\mathrm{APR}=$ abdominoperineal resection, $\mathrm{AR}=$ anterior resection, $\mathrm{CRT}=$ chemoradiation therapy, $\mathrm{pCR}=$ pathological complete response

Table 4:-Clinical versus pathological tumor and nodal staging $(\mathrm{n}=56)$

\begin{tabular}{|c|c|c|c|c|c|c|c|}
\hline \multirow[t]{2}{*}{ Baseline Staging } & \multicolumn{7}{|c|}{ Pathological Staging } \\
\hline & pT0 & pT1 & $\mathrm{pT} 2$ & $\mathrm{pT} 3$ & pT4 & $\begin{array}{c}\mathrm{pN} \\
\text { negative }\end{array}$ & $\begin{array}{c}\mathrm{pN} \\
\text { positive }\end{array}$ \\
\hline cT3 & $16(28.6 \%)$ & $10(17.8 \%)$ & $6(10.1 \%)$ & $12(21.4 \%)$ & - & - & - \\
\hline cT4 & - & $\begin{array}{c}1 \\
(1.8 \%)\end{array}$ & $\begin{array}{c}3 \\
(5.4 \%)\end{array}$ & $\begin{array}{c}4 \\
(7.1 \%)\end{array}$ & $\begin{array}{c}4 \\
(7.1 \%)\end{array}$ & - & - \\
\hline $\mathrm{cN}$ positive & - & - & - & - & - & $25(44.6 \%)$ & $14(25 \%)$ \\
\hline $\mathrm{cN}$ negative & - & - & - & - & - & $13(23.2 \%)$ & $\begin{array}{c}4 \\
(7.1 \%)\end{array}$ \\
\hline
\end{tabular}

Abbreviations: $\mathrm{c}=$ clinical (assessed by imaging); $\mathrm{P}=$ assessed pathologically

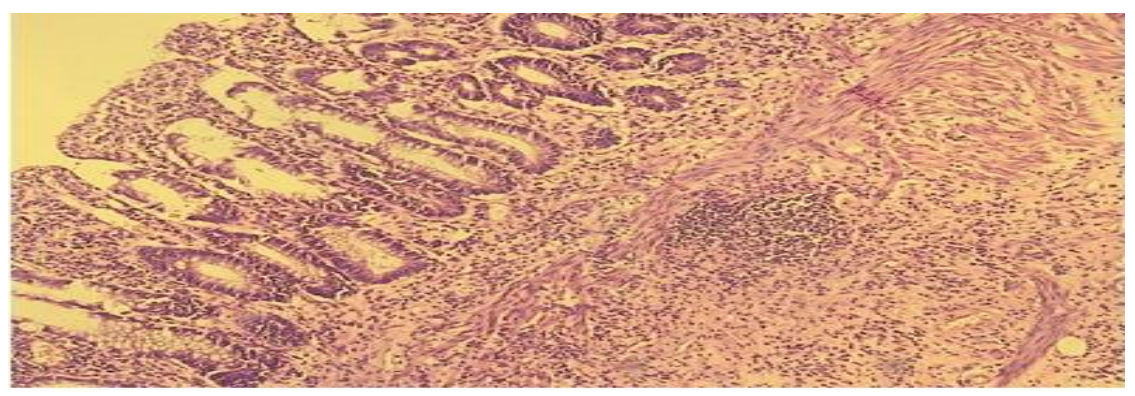

Figure 1:-Example of complete pathological response (H\&E x400)

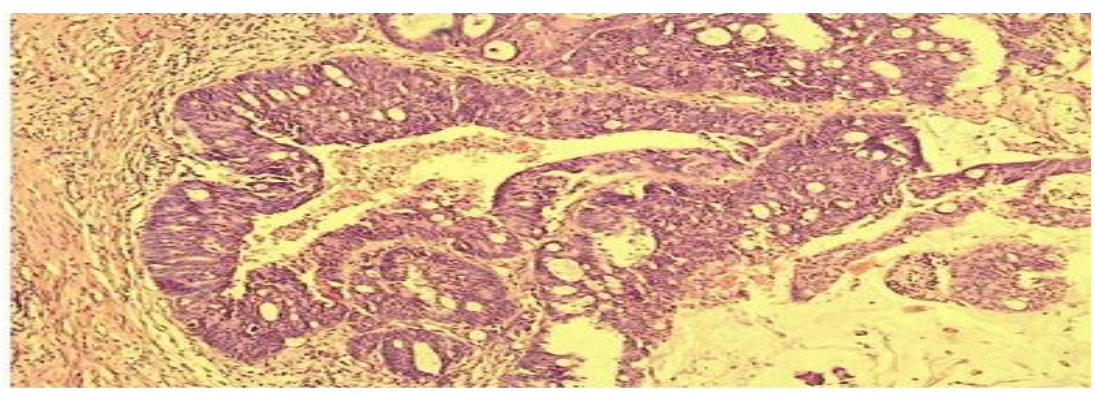

Figure 2:-Example of poor pathological response (H\&E x400)

\section{References:-}

1. Siegel R, Naishadham D, Jemal A. Cancer Statistics.CA Cancer J Clin 2013;63:11-30.

2. Sauer R, Becker H, Hohenberger W, Rodel C, Wittekind C, Fietkau R, et al. Preoperative versus postoperative chemoradiotherapy for rectal cancer. N Engl J Med 2004; 351:1731-40.

3. Guckenberger M, Saur G, Wehner D, Sweeney RA, Thalheimer A, Germer CT, Flentje H. Comparison of preoperative short- course radiotherapy and long-course radiochemotherapy for locally advanced rectal cancer. Strahlenther Oncol 2012; 188:551-57.

4. Peeters KC, Marijen CA, Nagtegaal ID, kranenbarg EK, Putter H, Wiggers T, et al. The TME trial after a median follow up of 6 years: increased local control but no survival benefit in irradiated patiets with resectable rectal carcinoma. Ann Surg 2007; 246:693-701.

5. Kapiteijn E, Marijne CA, Nagtegaal ID, Putter H, Steup WH, Wiggers T, et al. Preoperative radiotherapy combined with total mesorectal excision for resectable rectal cancer. N Engl J Med 2001;345:638-46

6. Gérard JP, Conroy T, Bonnetain F, Bouché O, Chapet O, Closon-Dejardin MT, et al. Preoperative radiotherapy with or without concurrent fluorouracil and leucovorin T3-4 rectal cancers: results of FFCD 9203. J Clin Oncol 2006; 24:4620-25. 
7. Rodel C, Liersch T, Herman RM, Arnold D, Reese T, Hipp M, et al. Multicnter phase II tial of chemradiation with oxaliplatin for rectal cancer. J Clin Oncol 2007; 25:110-17.

8. Rodel C, Arnold D, Becker H, Fietkau R, Ghadimi M, Graeven U, et al. Induction chemotherapy before chemoradiotherapy and surgery for locally advanced rectal cancer. Is it time for a randomized phase III tial? Strahlenther Onkol 2010; 180(12): 658-90.

9. Garcia-Aguilar J, Smith DD, Avila K, Bergsland EK, Chu P, Krieg RM, et al. Optimal timing of surgery after chemoradiation for advanced rectal cancer: preliminary results of a multicenter, nonrandomized phase II prospective trial. Ann Surg 2011; 254:97-102.

10. Habr-Gama A, Perez RO, Sabbaga J, Nadalin W, Sao Juliao GP, Gama-Rodrigues J, et al. Increasing the rates of complete response to neoadjuvant chemoradiotherapy for distal rectal cancer :results of a prospective study using additional chemotherapy during the resting period. Dis Colon Rectum 2009; 52:1927-34.

11. Glynne-Jones R, Grainger J, Harrison M, Ostler P, Makris A. Neoadjuvant chemotherapy prior to preoperative chemoradiation or radiation in rectal cancer: Should we be more cautious? Br J Cancer 2006; 94:363-71.

12. Schou JV, Larsen FO, Rasch L, Linneman D, Langoff J, Høgdal E, et al. Induction chemotherapy with capecitabine and oxaliplatin followed by chemoradiotherapy before total mesorectal excision in patients with locally advanced rectal cancer.Ann Oncol 2012; 23:2627-33.

13. Marechel R, Vos B, Polu M, Delaunoit T, Peeters M, Demetter P, et al. Short course chemotherapy followed by concomitant chemotherapy and surgery in locally advanced rectal cancer: a randomized multicentric phase II study. Ann Oncol 2012; 23:1525-30.

14. Fernandez-Martos C, Pericay C, Aparicio J, Salud A, Safont M, Massuti B, et al. Phase II, randomized study of concomitant chemoradiotherapy followed by surgery and adjuvant capecitabine plus oxaliplatin (CAPOX) compared with induction CAPOX followed by concomitant chemoradiotherapy and surgery in magnetic resonance imaging- defined, locally advanced rectal cancer: Grupo Cancer de Recto 3 study. J Clin Oncol 2010;28:859-65.

15. Chau YJ, Barbachano Y, Cunningham D, Oates JR, Brown G, Wotherspoon A, et al. Neoadjuvant capecitabine and oxaliplatin before chemoradiotherapy and total mesorectal excision in MRI-defined poor-risk rectal cancer :a phase 2 trial. Lancet Oncol 2010; 11(3):241-48.

16. Koeberle D, Burkhard R, Von Moos R, Winterhalder R, Hess V, Heitzman F, et al. Phase II study of capecitabine and oxaliplatin given prior to and concurrently with preoperative pelvic radiotherapy in patients with locally advanced rectal cancer. Br J Cancer 2008; 98 (7):1204-9

17. Garajova I, Di Girolamo S, de Rosa F, Corbelli J, Agostini V, Biasco G, et al. Neoadjuvant treatment in rectal cancer : actual status. Chemother Res pract. 2011; 2011:839742.

18. Common Terminology Criteria for Adverse Events (CTCAE), Version 4.0. Published: May 28,2009:http :// evs.nci.nih.gov/ftp1/CTCAE/ CTCAE_4.03_2010-06-22.Accepted 21 NOV 2011

19. Washington MK, Berlin J, Branton P, Bugart LJ, Carter DK, Fitzgibbons PL, et al. Protocol for the examination of specimens from patients with primary carcinoma of the colon and rectum. Arch Pathol Lab Med 2009; 133:1539-51.

20. Trakarnsanga A, Gönen M, Shia J, Nash GM, Temple LK, Guillem JG, et al. Comparison of tumor regression Grade Systems for locally Advanced rectal cancer After Multimodality treatment. JNCI J Natl Cancer Inst 2014;106 (10): dju248

21. American Joint Committee on cancer. AJCC Cancer staging Manual. $7^{\text {th }}$ ed. New York: Springer; 2010.

22. Hall MD, Schultheiss TE, Smith DD, Fakih MG, Kim J, Wong JY, et al. Impact of total lymph node count on staging and survival after neoadjuvant chemoradiation therapy for rectal cancer. Ann surg Oncol 2015; 22:58087.

23. Bosset JF, Collette L, Calais G, Mineur L, Maingon P, Radosevic-Jelic L, et al. Chemotherapy with preoperative radiotherapy in rectal cancer. N Engl J Med 2006; 355:1114-23.

24. Rodel C, Lierch T, Becker H, Fietkau R, Hohenberger W, Hothorn T, et al. Preoperative chemoradiotherapy and postoperative chemotherapy with fluorouracil and oxaliplatin versus fluorouracil alone in locally advanced rectal cancer : initial results of the German CAO/ARO/AIO-04 randomised phase 3 trial. Lancet Oncol 2012;13:679-87.

25. Zampino MG, Magni F, Leonardi MC, Petazzi E, Santoro L, Luca F, et al. Capecitabine initially concomitant to radiotherapy then perioperatively administered in locally advanced rectal cancer. Int J Radiat Oncol Biol Phys 2009; 75:421-27.

26. Cercek A, Goodman KA, Hajj C, Weisberger E, Segal NH, Stadler ZK, et al. Neoadjuvant chemotherapy first, followed by chemoradiation and then surgery in the management of locally advanced rectal cancer. JNCCN $2014 ; 4: 513-19$. 
27. Chau I, Brown G, Cunningham D, Tait D, Wotherspoon A, Norman AR, et al. Neoadjuvant capecitabine and oxaliplatin followed by synchronus chemoradiation and total mesorectal excision in magnetic resonance imaging - defined poor -risk rectal cancer. J Clin Oncol 2006;24:668-74.

28. Gao YH, Lin JZ, An X, Luo JL, Cai MY, Cai PQ, et al. Neoadjuvant sandwich treatment with oxaliplatin and capecitabine administered prior to, concurrently with, and following radiation therapy in locally advanced rectsl cancer. Int J Radiat Oncol Biol Phys 2014; 90:1153-60

29. Achele C, Cionini L, Lonardi S, Pinto C, Cordio S, Rosati G, et al. Primary tumor response to preoperative chemoradiation with or without oxaliplatin in locally advanced rectal cancer: Pathologic results of the STAR01 randomized phase III trial. J Clin Oncol 2011; $29: 2773-80$.

30. An X, Lin X, Wang FH, Goodman K, Cai PQ, Kong LH, et al. Short term results of neoadjuvant chemoradiotherapy with fluoropyrimidine alone or in combination with oxalipltin in locally advanced rectal cancer :a meta analysis. Eur JCancer 2013;49:843-51.

31. Calvo FA, Serrano FJ, Diaz-Gonzalez JA, Gomez-Esp M, Lozano E, Garcia R, et al. Improved incidence of pT0 downstaged surgical specimens in locally advanced rectal cancer (LARC) treated with induction oxaliplatin plus 5-fluorouracil and preoperative chemoradiation. Ann Oncol 2006; 17:1103-10.

32. Finn OL, Alice M, Benny VJ, et al.Capecitabine and oxaliplatin before,during, and after radiotherapy for highrisk rectal cancer.Clin Colorectal Cancer 2016;16:7-14

33. Zorcolo L, Rosman AS, Restivo A, Pisano M, Nigri GR, Fancellu A, et al. Complete pathologic response after combined modality treatment for rectal cancer and response after combined modality treatment for rectal cancer and long-term survival: a meta-analysis. Ann Surg Oncol 2012;19:2822-32

34. Bouzourene H, Bosman FT, Seelentag W,et al. Importance of tumor regression assessment in predicting the outcome of patients with locally advanced rectal carcinoma who are treated with preoperative radiotherapy. Cancer 2002;94:1121-30

35. Chapet O, Romestaing P, Mornex F, Souquet JC, Favrel V, Ardiet JM, et al. Preoperative radiotherapy for rectal adenocarcinoma: which are strong prognostic factors? Int J Radiat Oncol Biol Phys 2005; 61:1371-77.

36. Rodel C, Martus P, Papadoupolos T, Fuzesi L, Klimpfinger M, Fietkau R, et al. Prognostic significance of tumor regression after preoperative chemoradiotherapy for rectal cancer. J Clin Oncol 2005; 23: 8688-96. 